\title{
Multicarrier waveforms with I/Q staggering: uniform and nonuniform FBMC formats
}

\author{
Slađana Jošilo, Miloš Pejović, Branko Đorđević, Milan Narandžić and Slobodan Nedić
}

\begin{abstract}
Starting from the well-known uniform filter bank multicarrier (FBMC) format with Nyquist spectral shaping of frequency-limited orthogonal (FLO) subchannels and its dual time-frequency, time-limited orthogonal (TLO) form, we introduce an orthogonal frequency division multiplex of nonuniformly spaced subchannels with unequal width. The orthogonality conditions for nonuniform FBMC (Nu-FBMC) were evaluated through simulations using the 'extended orthogonal frequency division multiplexing (OFDM)' framework, as well as through analytical derivation in a rather pragmatic manner. The referent filter bank impulse responses were defined in frequency domain by straightforward aggregations of the pertaining uniform filter bank subchannels spectral shapes for FLO and by transforming the frequency domain of appropriately aggregated time-limited referent impulse responses of uniform TLO configuration. Nonsymmetrical spectral shaping of subchannels in the FLO case format has also been proposed. The spectral efficiency has been evaluated in the presence of nonlinear distortions caused by high power amplifier (HPA), and results have been given in accordance with power spectral density (PSD) constraints defined by IEEE 802.11a standard.
\end{abstract}

Keywords: Filter bank multicarrier (FBMC); Frequency-limited orthogonal (FLO); Nonuniform filter bank multicarrier (Nu-FBMC); Orthogonal frequency division multiplexing (OFDM); Time-limited orthogonal (TLO); Offset (or staggered) QAM (OQAM)

\section{Introduction}

With the ever increasing demand for higher wireless network throughput and number of supported users, efficient utilization of spectrum and development of effective means to overcome the co-channel interference have become a very important and challenging area of research. The cyclic prefix-based orthogonal frequency division multiplexing (CP-OFDM) as the most common and widely used multicarrier technique has many disadvantages in terms of the above mentioned requirements, which necessitates further improvement of the existing multicarrier techniques. One of the possible and quite promising solution is the usage of staggered modulation formats that sequentially, at $T / 2$ separated instants, transmit the in-phase (I or Re) and the quadrature (Q or Im) components of the M-ary quadrature amplitude modulation (M-QAM) data symbols of duration $T$, prevalently known as offset QAM (OQAM). This modulation format creates the basis for efficient utilization of spectrally shaped subchannels of the filter bank multicarrier (FBMC)

\footnotetext{
* Correspondence: nedics@uns.ac.rs

University of Novi Sad, Trg Dositeja Obradovića 6, Novi Sad, Serbia
}

aggregations, also known as OFDM/OQAM that can reduce the adjacent channel interference, at least without the presence of nonlinear distortions, whose significance has been discussed in [1] (The cosine-modulated multitone (CMT) therein is actually a form of OFDM/OQAM with odd-spaced subchannels). On the other side, this modulation format offers an additional degree of freedom, enabling suppression of the kindred co-channel interference based on the quasi-rectilinear constellation and the intrinsically real domain orthogonality by means of application of the minimum mean square error widely linear filtering (MMSE WLF) equalization [2-4]. Starting from the time-frequency duality between standard OFDM/OQAM formats and TLO multicarrier formats, we introduce uniform (even- and odd-spaced) FBMC-FLO and FBMC-TLO configurations. The frequency-limited orthogonal (FLO) and the time-limited orthogonal (TLO) denote the FBMC options with the nominal frequency domain and the time-domain limited subchannels' signaling elements, respectively, that are the orthogonal multicarrier systems where there is respective overlapping between adjacent subchannels in each of the subchannels. Although

\section{Springer}


the standard OFDM/OQAM can support prototype functions derived without such conceptualization, as is so called IOTA with identically shaped prototype impulse response and its Fourier transform [5], the paper considers more practical FLO prototype functions and its dual form named TLO becomes an extension of the OFDM/MSK approach therein.

In order to harmonize the advantages of using wider subchannels in terms of reduction of peak-to-average power ratio (PAPR) and the increase of spectral efficiency in situations when predetermined power spectral density (PSD) masks have to be obeyed, the need for a modification arises which would enable the utilization of subchannels with differing widths within scattered frequency bands (white zones). At the same time, it is important to be able to separate the adjacent channels by relatively narrow frequency guard intervals. This, in particular, relates to the available frequency gaps in the targeted private mobile radio (PMR) applications, the TV white spaces, and the wireless system bandwidths unoccupied by the primary users. This goal could be achieved, in principle, by adopting widely explored and quite well-studied nonuniform filter bank configurations (Nu-FBMC) known and used for source coding applications [6]. In particular, channelization approach proposed in [7] could be used; but due to specific orthogonality conditions for the data transmission, we have chosen a more pragmatic approach. Although the latter relies on spectral aggregation of uniformly spaced subchannels within the frequency-disjoined subbands, with the goal of their near-perfect reconstruction, its specialization to nonuniform data transmission related structures, in particular those of the TLO-type, turned out to be not that straightforward.

With appropriate modification of the uniform FLO and TLO configurations, we formed the nonuniform filter banks (Nu-FBMC) with non-equal subchannel width and with non-equidistant central frequencies by using the method of aggregation of the respective frequency- or time-domain shapes. The orthogonality conditions are analytically derived and confirmed through computer simulations for both the Nu-FBMC-FLO and the $\mathrm{Nu}$ FBMC-TLO configurations. For this purpose, we have used the framework of extended OFDM [8]. We also experimented and came up with the idea and confirmation of orthogonality conditions for the asymmetrical roll-off factors (in frequency domain) primarily to better control the latency inherent to the FBMC format. This will also allow for the multiplexing of unequally wide subchannels of FBMC waveforms belonging to different users without the need for an introduction of the frequency guard bands in the downlink (DL) direction of transmission.

After highlighting the time-frequency duality between uniformly spaced FBMC FLO and TLO formats in
Section 2, the use of the extended OFDM format is reproduced in Section 3, along with hints to some critical aspects regarding the need for oversampling and equalization. The method of aggregation, the uniformly spaced subchannels, and the related derivation of nonuniform filter bank orthogonality conditions are dealt in Section 4. In Section 5, the asymmetrical subchannel spectral shaping is introduced. The similar aggregation procedure is applied to the nonuniform TLO format in Section 6, but in time domain by means of the adequate positioning of their spectral representations along the frequency. In Section 7, we presented the comparison of CP-OFDM and FBMC (FLO/TLO) formats in context of spectral and power efficiency and PAPR reduction. A brief comparison with conventional approaches of the nonuniform filter bank design in Section 8 is followed by the conclusions in the last Section 9.

\section{Uniformly spaced TLO-MC and FBMC}

The standard FBMC based on OFDM/OQAM formats with frequency domain shaping could be conveniently termed as the frequency-limited orthogonal approach. The relations between FLO and the TLO [9] multicarrier formats can be established through their time-frequency duality, as illustrated in Figure 1. The shape of baseband FLO spectrum and the TLO pulse is controlled by the roll-off factor. For 0\% roll-off, TLO uses rectangular pulse of length $T / 2$; while for the $100 \%$ roll-off case, the pulse has the form of the half-cosine function of the length $T$. The latter corresponds to the conventional minimum shift keying (MSK) modulation format, where the I/Q staggering pattern shown in Figure 2 is implied for all the orthogonal referent pulse shapes whose subchannels' spectra overlap.

The designations of TLO and FLO are meant to be applied to each subchannel individually; but, as seen from the waveforms' time-frequency representation and their positioning, the absence of their mutual overlapping in both domains is not implied.

The implementation of TLO and FLO-FBMC formats, both in the uniform and nonuniform configurations, is considered in Section 3 through the extended OFDM concept that is essentially a method for designing the signaling pulse-shape in frequency domain.

\section{The extended OFDM framework}

The extended OFDM framework is proposed in FP7 PHYDYAS project for multichannel filtering [8] and corresponds to the frequency-domain implementation of the computationally efficient overlap-and-add method ${ }^{a}$ [10]. The modulator performs separate filtering of every subchannel with $2 K-1$ samples $\left(\hat{G}_{n, k}^{(K-1)}, \hat{G}_{n, k}^{(K-2)} \cdots \hat{G}_{n, k}^{(0)} \cdots\right.$ $\hat{G}_{n, k}^{(K-2)}, \hat{G}_{n, k}^{(K-1)}$ ) of filter transfer function $\hat{G}_{n, k}(\omega)$, in a 

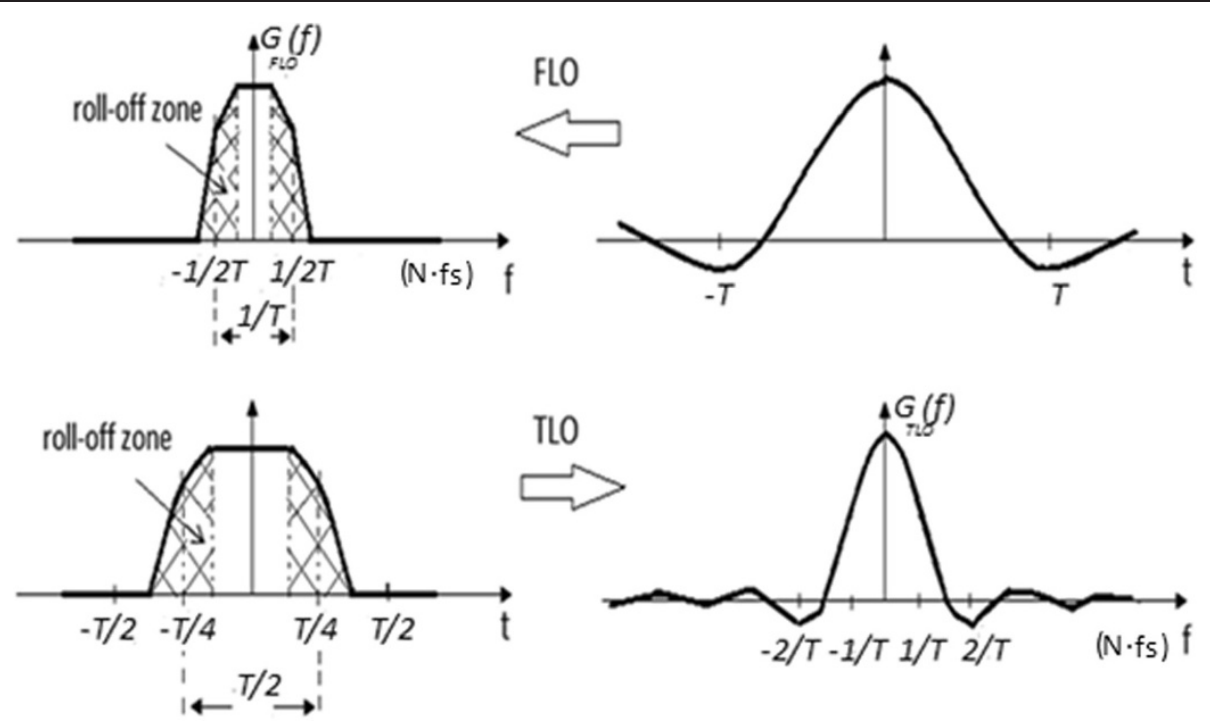

Figure 1 Time-frequency duality between the TLO and FLO formats, $N$ is the number of samples and $f s$ is sampling frequency.

form of weighting according to the scheme from Figure 3a. For data sequence of length $M$, the modulator uses $K \cdot M$-point inverse fast Fourier transform (IFFT), $K$ being the overlapping factor of the pertinent FBMC referent impulse responses in number of $T$ intervals. The result of subchannel filtering is obtained by $T / 2$ shifted subsequent outputs and their summations. The first and second indexes, $n$ and $k$ of transfer function $\hat{G}_{n, k}(\omega)$, are related to $T / 2$ instant number and subchannel central frequency, respectively. The superscript $j$ added in $\hat{G}_{n, k}^{(j)}$ indicates $j$-th sample of the subchannels transfer function $\hat{G}_{n, k}(\omega)$.

Figure 3a,b shows two nonadjacent and nonoverlapped subchannels with indexes $k$ and $k+2$. Subchannel with index $k+1$ overlaps with both; orthogonality is provided by the usage of real inputs of the IFFT for $k$ and $k+2$ and imaginary inputs for $k+1$, or vice versa.

The transposed configuration of the receiver part is shown in the block diagram of Figure $3 b$, whereby the $2 K-1$ samples of the $K \cdot M$-long FFT outputs are weighted with the same subchannel referent filter transfer function coefficients as in transmitter and summed to produce the subchannel outputs at $T / 2$ intervals.

The OFDM/OQAM application uses square-rootraised-cosine (SRRC) transfer function with predefined roll-off factor, while the oversampling factor is 2 (the real and imaginary parts of a complex QAM symbols are transmitted at $T / 2$ instants). The filter banks use either even-spaced (even multiples of $1 / 2 T$ frequency) or odd-spaced (odd multiples of $1 / 2 T$ frequency) filter bank arrangements, as shown in Figure 4. The subchannels' spectral shape is drawn as having 'linear' roll-off regions just for convenience.

The separation between the peaks of the channel SRRC transfer function with $100 \%$ roll-off factor is $1 / T$, corresponding to the inverse of the QAM symbol rate. The frequency shift between even and odd subchannels' central frequencies is $1 / 2 T$. Each of the individual spectral shapes $\hat{G}_{n, k}(\omega)$, shown by triangles in Figure 4 , contents $2 K-1$ equidistant samples $\hat{G}_{n, k}^{(j)}(\omega)$, as shown in Figure 3a,b.

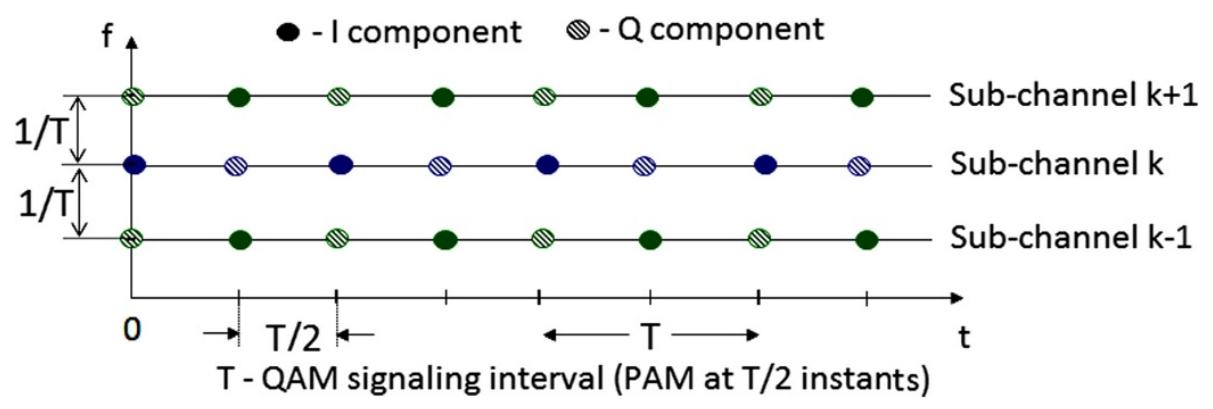

Figure 2 Signaling of I and $\mathrm{Q}$ parts in staggered modulation format. 


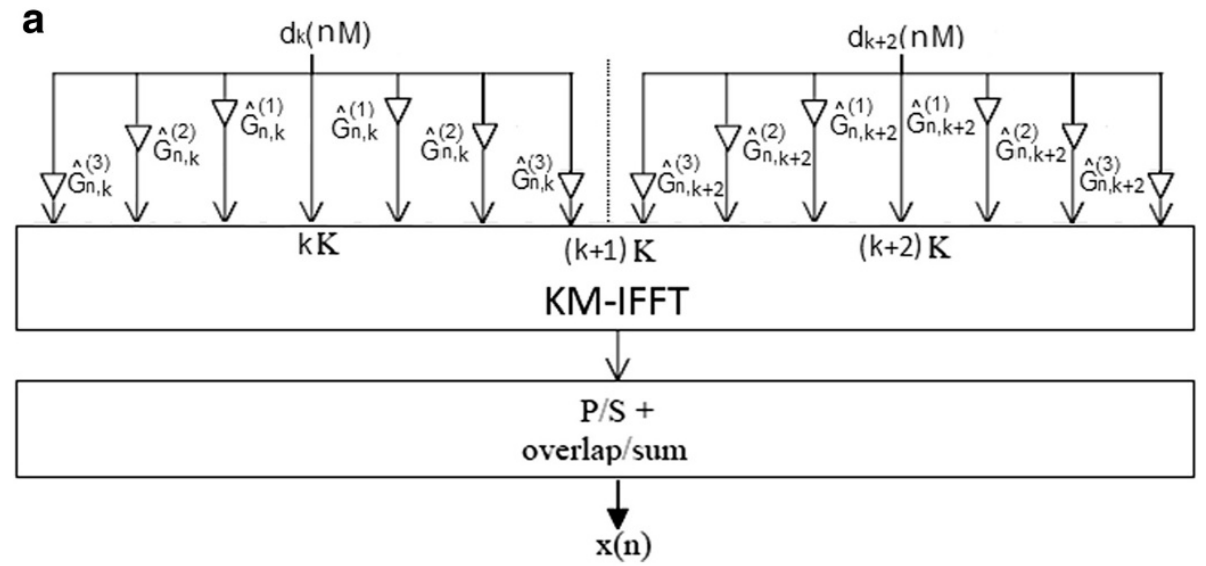

b
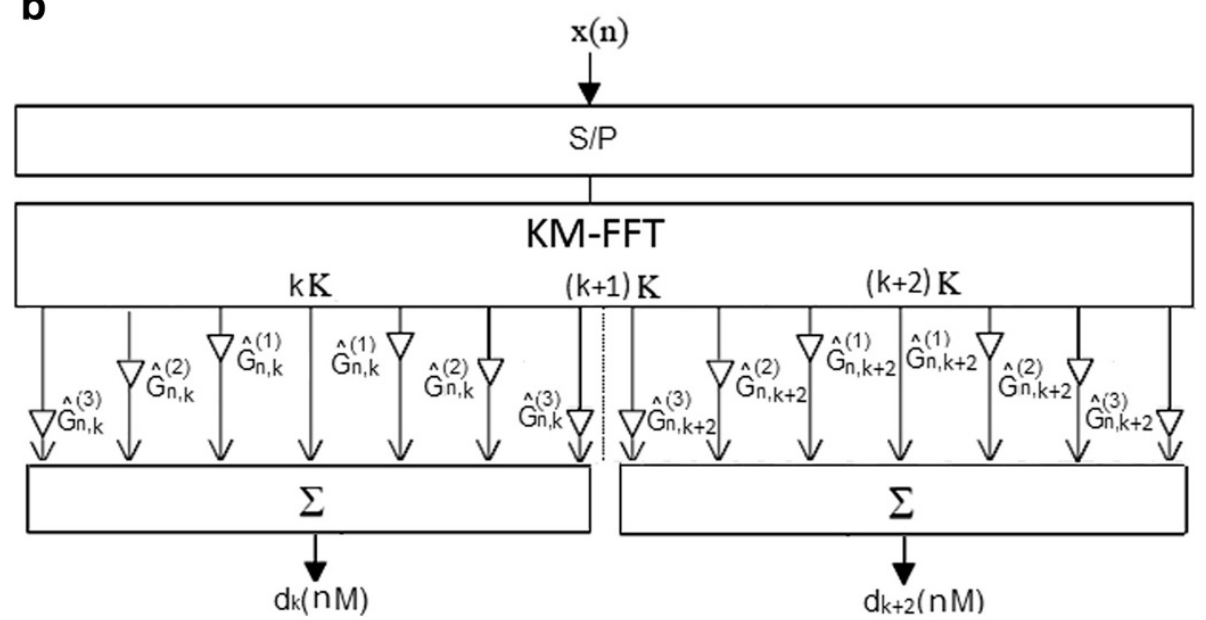

Figure 3 The frequency-domain configuration. (a) The frequency-domain modulator configuration for overlapping factor $K=4$. (b) The frequency-domain demodulator configuration for overlapping factor $K=4$.

The conditions for achieving (near) perfect orthogonality are arrangement dependant. For the even filter banks and the particular subchannel, the real and imaginary (including phase shift $j$ ) parts of QAM symbols are fed to the $K \cdot M$-point IFFT inputs sequentially at even and odd $T / 2$ instants. The order of the real and imaginary parts staggering is reversed on their adjacent subchannels. The I/Q staggering pattern shown in Figure 2 applies for this case. For the odd filter banks, the Re and $j \cdot I m$ parts of
QAM symbol are brought to the $K \cdot M$-point IFFT inputs (sent in all subchannels) at $T / 2$ instants as purely real or purely imaginary values.

\section{Aggregating subchannels for the Nu-FBMC-FLO format}

Direct extension of the uniform filter bank towards the nonuniform filter bank configuration, for the FLO case, is to simply aggregate the subchannels of the even, odd, or

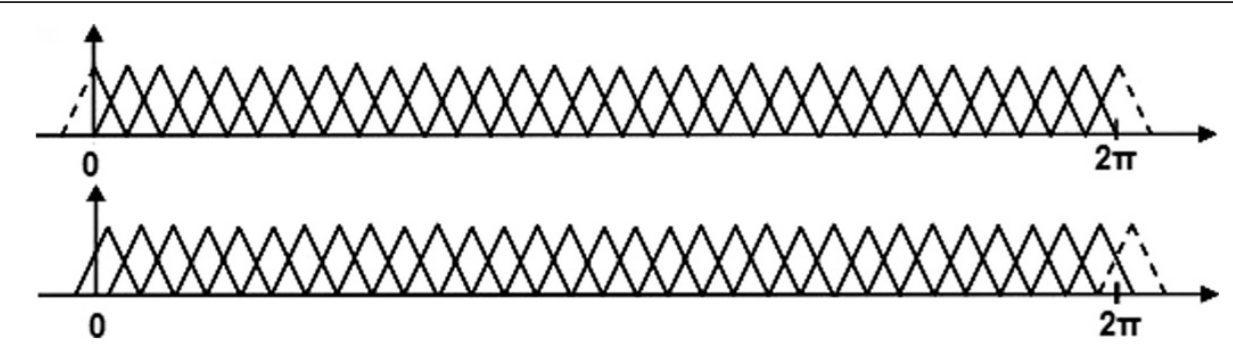

Figure 4 The even (upper part) and odd (lower part) uniform filter banks, $M=32$. 
combined uniform filter banks. Next, we propose the construction of nonuniform configurations and afterwards, we study the orthogonality conditions in terms of the Re and $j \cdot I m$ signaling instants and their relationship in the I/Q staggering pattern. For that purpose, we retain the roll-off region spectral symmetry from the uniform filter bank arrangements shown in Figure 4, with the frequency axis italicized by sampling frequency $f_{s}=M / T$, where $M$ is the number of subchannels, and $T$ is the duration of QAM interval.

\subsection{Concept of aggregation and selected configurations}

The $K \cdot M$-long vectors belonging to equidistantly spaced (real and positive) subchannel samples, among which there are generally up to $2 K-1$ non-zero values, are denoted by $\hat{G}_{n, k}$, with $k=0,1,2, \ldots, K M-2, K M-1$, then the wider and generally nonuniformly spaced subchannel samples can be defined as follows:

$$
s C h=\sqrt{\sum_{k=x}^{y} \hat{G}_{n, k}^{2}}
$$

where $x$ and $y$ define the range of subsequent (non-zero) uniform channels. For example, from the odd-spaced uniform filter bank with 32 uniform subchannels, the following arrangement of the non-equidistant subchannels can be made:

$$
\begin{array}{r}
\mathrm{sCh} 1 \mathrm{p}=\sqrt{\sum_{k=0}^{7} \hat{G}_{n, k}^{2}}, \mathrm{sCh} 2 \mathrm{p}=\sqrt{\sum_{k=8}^{11} \hat{G}_{n, k}^{2}}, \\
\mathrm{sCh} 3 \mathrm{p}=\sqrt{\sum_{k=12}^{13} \hat{G}_{n, k}^{2}}, \mathrm{sCh} 4 \mathrm{p}=\sqrt{\sum_{k=14}^{14} \hat{G}_{n, k}^{2}} \text {, as shown }
\end{array}
$$
in Figure 5. Due to DFT properties of each primary channel, $p$, with positive frequency below $f_{s} / 2$, will have its spectral counterpart at negative frequency (above $f_{S} / 2$ ) which is denoted with $m$ (mirror).

If the original subchannels in uniform arrangements have roll-off factor of $100 \%$ in the wider subchannels, it becomes progressively reduced to $50 \%, 25 \%$, and $12.5 \%$ by the effective doubling of subchannels' bandwidth. For aggregated channels, the signaling intervals are correspondingly halved as can be inferred from the illustration in Figure 5. The orthogonality conditions between the subchannels formed in this way are reduced to the ones of the uniform FBMC as shown in Section 4.2. The sum of the vectors pertinent to the nonuniform subchannels is fed to the input of a $K \cdot M$-size IFFT in the same way as in Figure $3 \mathrm{a}$, and similarly reproduced at the output of demodulator by weighting/summing them at the $K \cdot M$ size FFT output, as it was the case in Figure 3b.

Sequencing of the real and complex QAM symbol parts takes place at the beginning and the half of the corresponding QAM symbol intervals. The sequencing strategy generally differs from the ones used for uniform structures and has been empirically determined to be as follows: if particular subchannel, by its replication along the frequency axis, produces an odd uniform filter bank, its signaling always uses either Re or $j \bullet I m$. If, however, the aggregated subchannel forms an even uniform filter bank, then interchangeably, Re and $j \cdot \mathrm{Im}$ are brought to $K \cdot M$-IFFT block, so that the adjacent (nonuniformly spaced) subchannels at the same time instant are purely real and purely imaginary. For the odd nonuniform filter bank arrangement of Figure 5a, the signaling of QAM parts is shown in Figure 5b. Alternatively, sCh1p, 3p, $4 \mathrm{~m}$, and $\mathrm{sCh} 2 \mathrm{~m}$ can transmit $j \cdot \mathrm{Im}$ part, while remaining subchannels transmit the Re part. In all the figures, the Re part is marked by a square and the $j \cdot$ Im part by a diamond.

If nonuniform bank is formed from an odd uniform filter bank, its subchannels will be odd as well. However, the further aggregation of the first subchannel with its mirror forms the even subchannel if, e.g., the sCh1p and sCh1m (from Figure 5a) were merged according to (1), then the central (DC) subchannel would correspond to an even-spaced FBMC, as illustrated in Figure 6. It should be obvious that the signaling rate in new/aggregated sCh1 is doubled with respect to the previous one, and that, the time between the two (consecutive) signaling moments corresponds to the half of the previous symbol duration. Notice that subchannels $4 \mathrm{~m}, 3 \mathrm{~m}$, and sCh2m have switched $\operatorname{Re}$ and $j \cdot I m$ parts in order to ensure an alternate sending of Re and $j \bullet I m$ parts in adjacent subchannels.

For the derivation of a nonuniform FBMC configuration by a uniform filter bank subchannels aggregation, only integer ratios of the extended and the original bandwidths have been considered. In order to keep the integer ratios of the produced nonuniform subchannels bandwidths and to still have every $\mathrm{Nu}$-FBMC subchannel belonging to either the even or the odd extended uniform FBMC 'category', certain subchannels have to be defined explicitly, or by a combination of segments of both the even and the odd (referent) uniform FBMC arrangements. Figure 7 illustrates such a situation. Here, the subchannels 2 and 3 contain 4.5 uniform subchannel lengths. Consequently, the roll-off factors (relative to the subchannels' Nyquist frequency) are $11.11 \%$. Although the signaling intervals of the subchannel 4 are not commensurate with the signaling intervals of the adjacent subchannels, the orthogonality conditions are fulfilled with the sequencing of QAM parts according to Figure 6. This indicates that the non-integer signaling speeds might be possible, although it is likely that it could be impractical for the symbol synchronization.

\subsection{Analytical derivation of orthogonality conditions}

The rather pragmatic approach to formal verification of orthogonality conditions in the case of nonuniform FLO and TLO will rely on orthogonality conditions for the related uniform filter banks.

Conditions for the intrinsic orthogonality of uniform FLO system expressed in time-domain ([9], reference [1] therein) are as follows: 


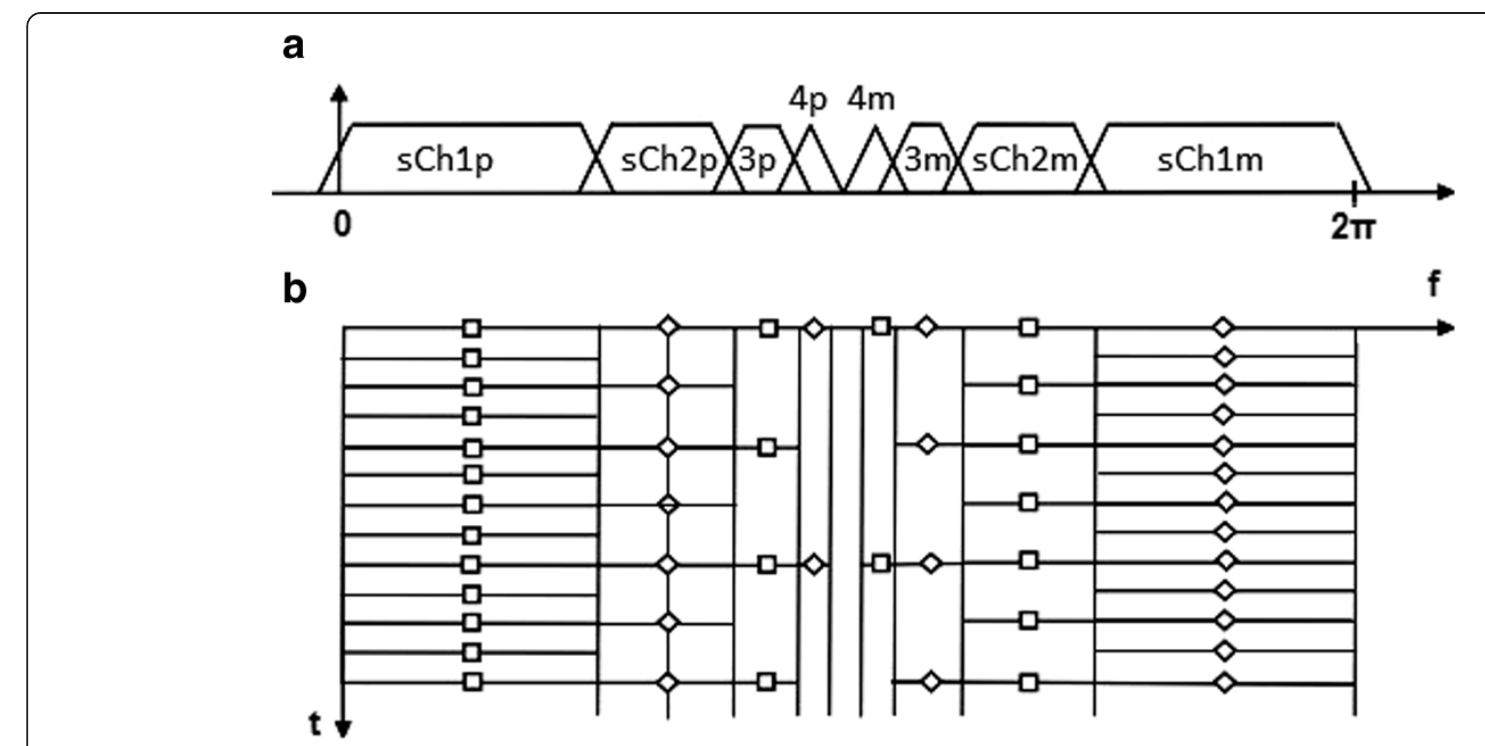

Figure 5 The nonuniform FBMC arrangement and the example of sequencing. Nonuniform FBMC arrangement made from odd uniform FBMC (a) and the example of sequencing the QAM I and Q parts in odd-Nu-FBMC (b).

$$
\operatorname{Re}\left\{\int_{-\infty}^{\infty} \hat{g}_{n, k}(t) \hat{g}_{m, l}^{*}(t) d t\right\}= \begin{cases}1 & k=l, n=m \\ 0 & \text { other }\end{cases}
$$

where the first and second indices are related to $\frac{T}{2}$ signaling interval and subchannel central frequency, respectively. The function $\hat{g}_{n, k}(t)$ is given by the following:

$$
\hat{g}_{n, k}(t)=f\left(t-\frac{n T}{2}\right) \exp \left(j \frac{2 \pi k}{T} t+j \phi_{n, k}\right)
$$

where

$$
\phi_{n, k}=\left\{\begin{array}{ccc}
\frac{\pi}{2} & n+k & \text { odd } \\
0 & n+k & \text { even }
\end{array}\right.
$$

$T$ is QAM symbol period and $f(t)$ is the impulse response of the square-root Nyquist filter with transfer function $F(\omega)$.

Relations between orthogonality conditions in time and frequency domains can be established according to Plancherel's theorem (extended Parseval's):

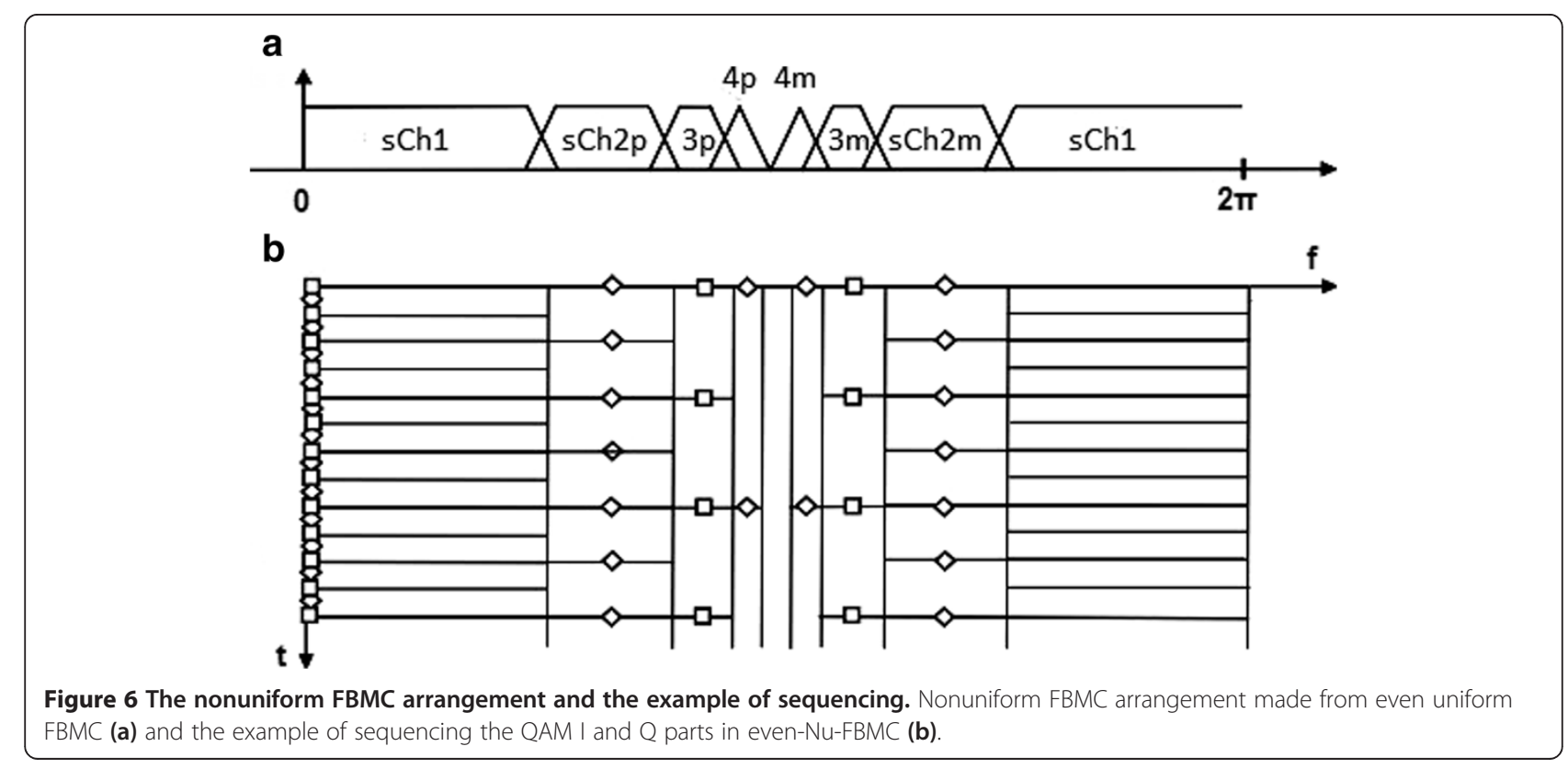




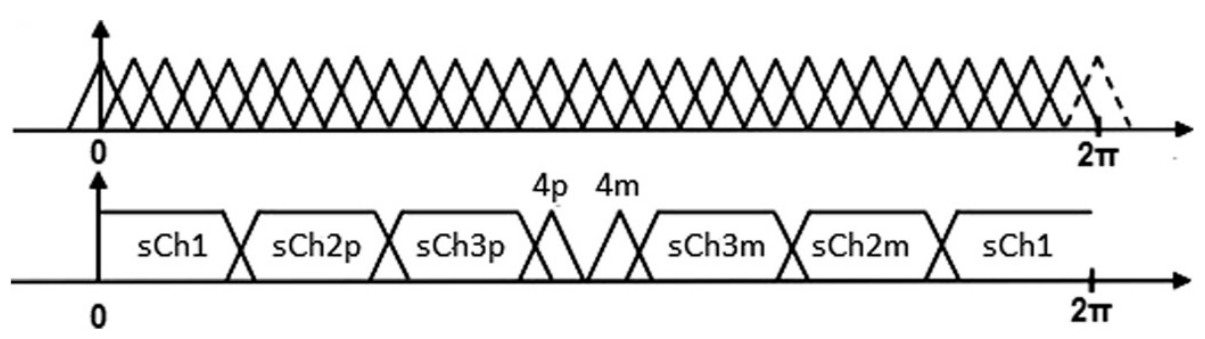

Figure 7 Nonuniform FBMC arrangement derived from even uniform FBMC.

$$
\int_{-\infty}^{\infty} \hat{g}_{n, k}(t) \hat{g}_{m, l}^{*}(t) d t=\frac{1}{2 \pi} \int_{-\infty}^{\infty} \hat{G}_{n, k}(\omega) \hat{G}_{m, l}^{*}(\omega) d \omega
$$

Following this identity, the derivation of the orthogonality conditions for the uniform (FBMC-)TLO system in [9] uses the Fourier transform of $\hat{g}_{n, k}(t), \hat{G}_{n, k}(\omega)$. The resulting formulation of orthogonality conditions in frequency domain therefore becomes as follows:

$$
\begin{aligned}
& \operatorname{Re}\left\{\int_{-\infty}^{\infty} \hat{G}_{n, k}(\omega) \hat{G}_{m, l}^{*}(\omega) d \omega\right\} \\
& = \begin{cases}1 & k=l, n=m \\
0 & \text { other }\end{cases}
\end{aligned}
$$

where

$$
\hat{G}_{n, k}(\omega)=F\left(\omega-\frac{2 \pi k}{T}\right) \exp \left(-j \frac{n T}{2} \omega+j \phi_{n, k}\right)
$$

For the straightforward design of the nonuniformly spaced subchannels by aggregation of the uniform FBMC subchannels transfer functions, the orthogonality conditions of the former can be reduced to conditions of the latter, as shown in Figure 8.

In Figure 8b, the subchannels $K$ and $L$ are described by (8) and (9), respectively.

$$
\begin{aligned}
& \hat{G}_{N, K}(\omega)=\sqrt{\sum_{i^{\prime}=i}^{0} \hat{G}_{n, k-i^{\prime}}^{2}(\omega)} \\
& \hat{G}_{M, L}(\omega)=\sqrt{\sum_{j^{\prime}=0}^{j} \hat{G}_{m, l+j^{\prime}}^{2}(\omega)}
\end{aligned}
$$

By placing the newly generated transfer functions into (6), it follows

$$
\begin{aligned}
& \operatorname{Re}\left\{\int_{-\infty}^{\infty} \hat{G}_{N, K}(\omega) \hat{G}_{M, L}^{*}(\omega) d \omega\right\}= \\
= & \operatorname{Re}\left\{\int_{-\infty}^{\infty} \sqrt{\sum_{i^{\prime}=i}^{0} \hat{G}_{n, k-i^{\prime}}^{2}(\omega)}\left(\sqrt{\sum_{j^{\prime}=0}^{j} \hat{G}_{m, l+j^{\prime}}^{2}(\omega)}\right)^{*} d \omega\right\}
\end{aligned}
$$

Since the complex conjugation (*) can be brought under the square root as well as under the squaring operation, and since this is applicable $(c 1+c 2)^{*}=c 1 *+$ $c 2 *$, the following expression is obtained:

$$
\begin{gathered}
\operatorname{Re}\left\{\int_{-\infty}^{\infty} \sqrt{\hat{G}_{n, k-i}^{2}(\omega)+\cdots+\hat{G}_{n, k-1}^{2}(\omega)+\hat{G}_{n, k}^{2}(\omega)} .\right. \\
\left.\sqrt{\hat{G}_{m, l}^{* 2}(\omega)+\cdots+\hat{G}_{m, l+(j+1)}^{* 2}(\omega)+\hat{G}_{m, l+j}^{* 2}(\omega)} d \omega\right\}
\end{gathered}
$$

Due to spectral confinement to widths $1 / T$ from all of the products situated under the root square operation, only $\hat{G}_{n, k}^{2}(\omega) \hat{G}_{m, l}^{* 2}(\omega)$ is different from zero, so the orthogonality conditions are reduced to those of the uniform FLO FBMC format, i.e., as follows:

$$
\begin{aligned}
\operatorname{Re}\left\{\int_{-\infty}^{\infty} \hat{G}_{N, K}(\omega) \hat{G}_{M, L}^{*}(\omega) d \omega\right\} & =\operatorname{Re}\left\{\int_{-\infty}^{\infty} \hat{G}_{n, k}(\omega) \hat{G}_{m . l}^{*}(\omega) d \omega\right\} \\
& = \begin{cases}1 & k=l, n=m \\
0 & \text { other }\end{cases}
\end{aligned}
$$

\section{Using asymmetrical subchannels for the Nu-FBMC-FLO format}

The relatively low roll-off factors resulting from the uniform FBMC aggregation might be unfavorable for at least two reasons: increased transmission delay due to lengthening of the referent impulse response and related increase of the PAPR, since the latter depends on both the number of subchannels and their impulses' length. In order to solve these problems, it would be of interest to keep the $\mathrm{Nu}$-FBMC subchannels' roll-off factors as large as possible, while retaining the same flexibility in assigning the subchannel bandwidths. This is possible by a direct modification of the subchannels' frequencydomain shapes, as shown in Figure 9. Here, we again start from the odd uniform filter bank arrangement, define a set on nonuniform subchannels by the aggregation, and then maximally increase the roll-off factors. The increase is more significant if the adjacent subchannels are wider.

The transmission I/Q sequencing is the same as in Figure 6, only extended to the increased number of subchannels, since the DC subchannel is inherently even- 

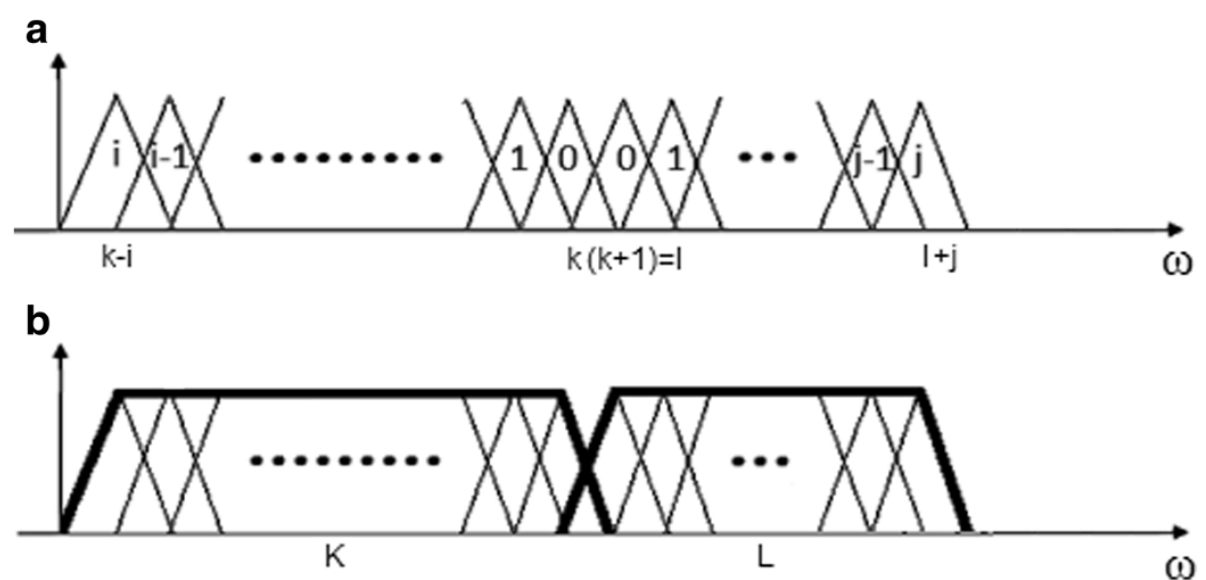

Figure 8 Spectral aggregation examples. (a) Uniform configuration. (b) Corresponding nonuniform configuration.

spaced, and other subchannels are odd-spaced. The maximal roll-off factor is $100 \%$ and the minimal is $50 \%$, evaluated with respect to the subchannels' respective Nyquist frequency. The simulation check has confirmed the intrinsic orthogonality of the Nu-FBMC format: as long as the overlapping regions are Nyquist-like symmetrical, the orthogonality is preserved no matter what the relative roll-off factors on the two sides might be.

In all analyzed examples evaluated by the computer simulation, we essentially had a zero frequency gap between the positive and negative frequencies (spectral parts), made by omitting only one subchannel. For easier separation and filtering-out of the spectral images in the process of low-pass filtering at the transmitter and suppression of spectral images aliasing in the receiver (before modulation to the RF carrier frequency and after demodulation), a wider gap can be obtained by only appropriately increasing the base-band processing sampling frequency.

One very important advantage of using roll-off factors in the range from $100 \%$ to $50 \%$ is the possibility to use relatively small 'oversampling' factors $K$ so that the increase in length of the (I)FFT $\mathrm{FM}_{K M}$ with respect to hypothetically used (I)FFT and polyphase networks (PNs) is rather low and much smaller than in the case of the same nonuniform arrangement with frequency domain weighted overlap-and-add method. The plots shown in Figures 10 and 11 illustrate the spectral arrangement of subchannels in the symmetrical and asymmetrical cases and their corresponding time-domain representation. Rolloff factors of subchannels in symmetrical arrangement are $100 \%, 50 \%, 50 \%, 25 \%$, 25\%, $12.5 \%$, and $6.25 \%$, respectively, starting from the narrowest (the rightmost) subchannel to the central (DC) subchannel (Figure 10a). In the case of asymmetrical arrangement, roll-off factors of subchannels are $100 \%$ to $100 \%, 100 \%$ to $50 \%, 50 \%$ to $50 \%$, $50 \%$ to $25 \%, 25 \%$ to $25 \%, 25 \%$ to $12.5 \%$, and $12.5 \%$ to $12.5 \%$, respectively, starting from the narrowest (the rightmost) subchannel to central (DC) subchannel (Figure 11a) (used notation ' $x \%-y \%$ ' denotes 'roll-off factor of right edge of subchannel-roll-off factor of left edge of subchannel'). In general, as mentioned before, the shorter impulse responses can potentially contribute to the reduction of FBMC signal PAPR, and thus reduce the spectral spread-

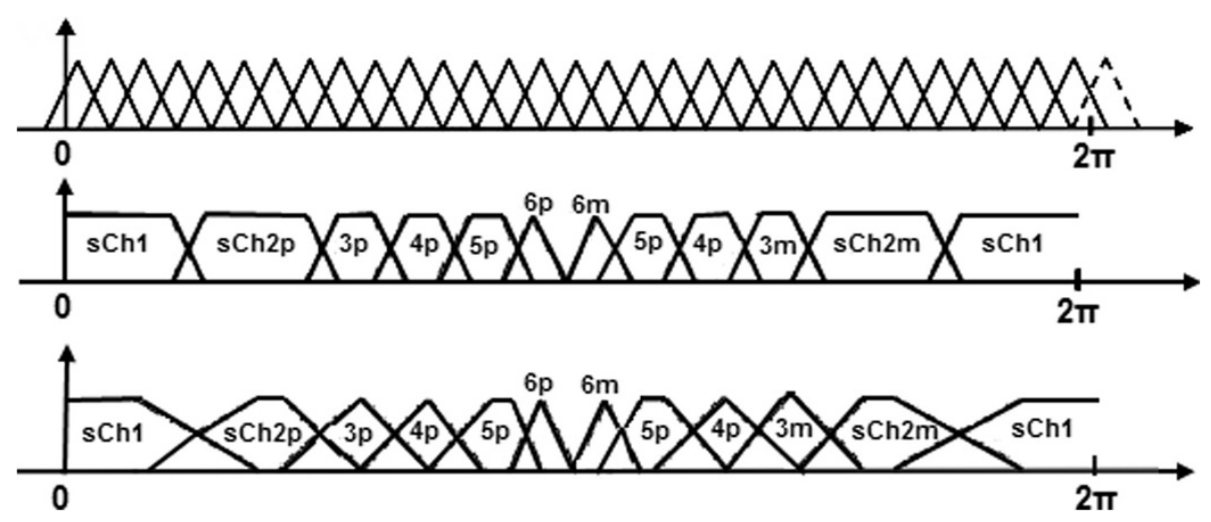

Figure 9 Nu-FBMC with asymmetrical subchannels' spectra. 


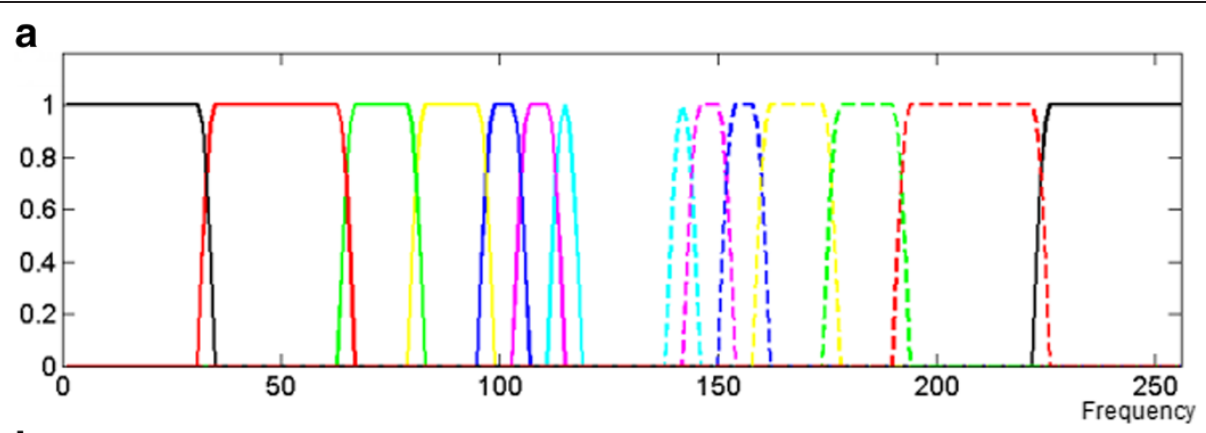

b

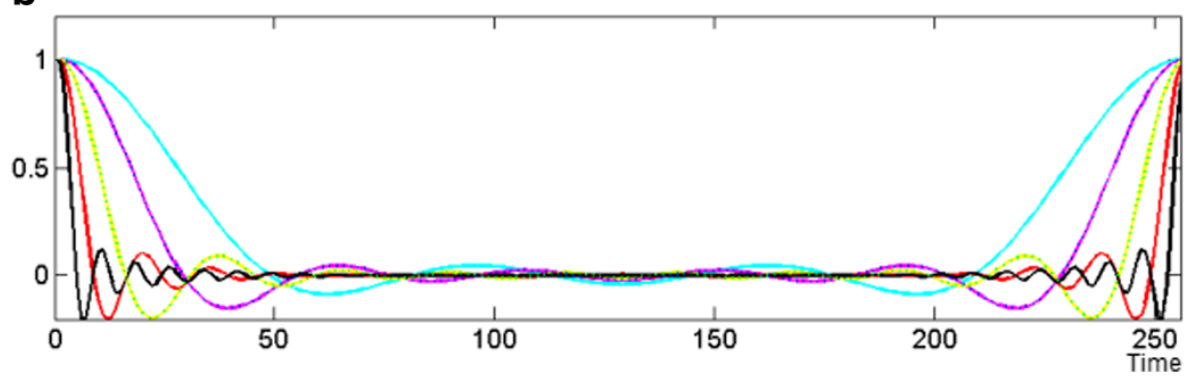

Figure 10 Nu-FBMC-FLO arrangements and representations. Nu-FBMC-FLO arrangements with symmetrical spectra (a) and corresponding time-domain representations (b)

out in case of nonlinear amplification. This is illustrated and somewhat corroborated by the simulation results in Section 7.

Figure 12 illustrates the matching of transmitted and received data (marked with red circles and green dots, respectively) for the even Nu-FBMC arrangement with symmetrical and asymmetrical spectra depicted in Figure 10a and Figure 11a.

Diagrams from Figure 12 are zoomed to the range \pm 0.02 around referent value \pm 1 for clarity of illustration, since the received symbols are insignificantly scattered around the nominal values in all considered cases.

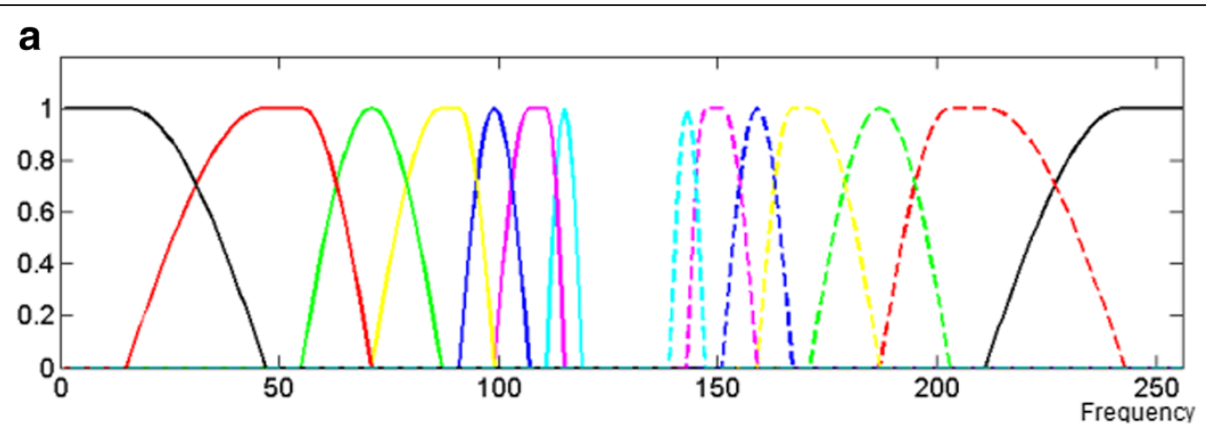

b

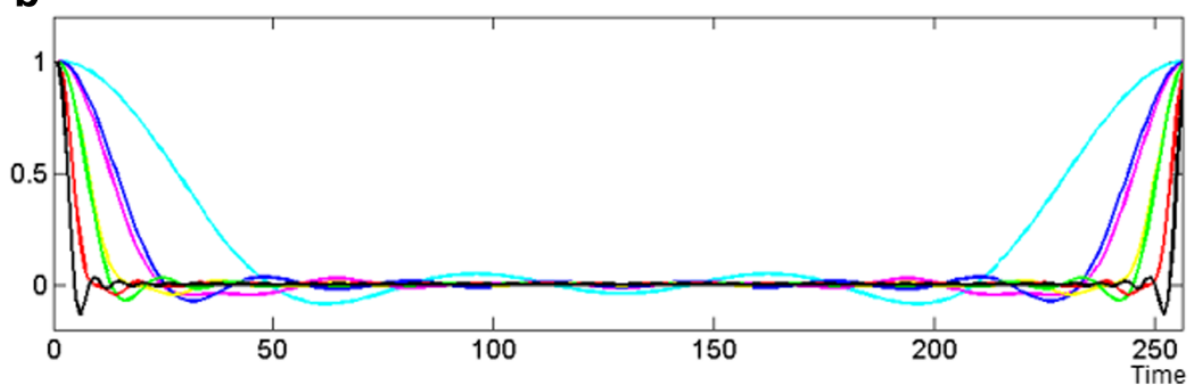

Figure 11 Nu-FBMC-FLO arrangements and representations. Nu-FBMC-FLO arrangements with asymmetrical spectra (a) and corresponding time-domain representations (b). 

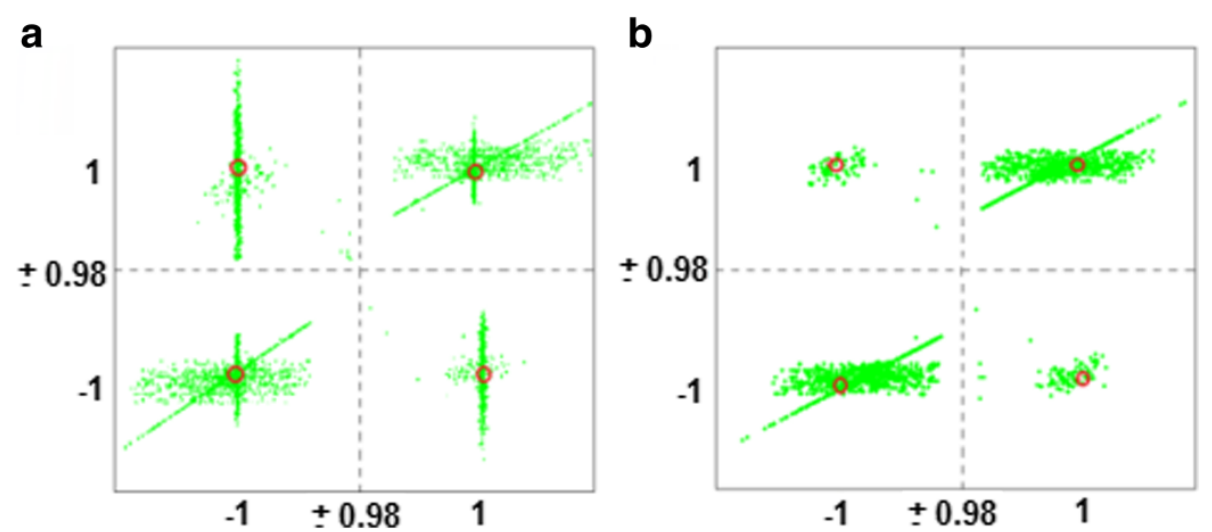

Figure 12 Matching between transmitted (red circles) and received data (green dots). (a) Symmetrical spectra, MSE $=8.1 \cdot 10^{-4}$; (b) asymmetrical spectra, MSE $=8.3 \cdot 10^{-4}$. The squares have sizes of 0.04 and cover the area around the nominal QAM real and imaginary values of \pm 1 .

While there is no noticeable difference in the data reconstruction accuracy (MSE $\sim 8.1^{\prime \prime} 10^{-4}$ and MSE $\sim 8.3^{\prime \prime} 10^{-4}$ for symmetrical and asymmetrical cases, respectively), by comparing the subchannels impulse responses in Figure 10b and Figure 11b, it can be seen that they are much shorter in the latter case, in particular for the widest subchannels with the largest relative increase of roll-off factors.

\section{Aggregating the time-domain signaling pulses for the Nu-FBMC-TLO format}

Similarly, as the frequency-domain representations of the subchannels spectra were produced by aggregation of the uniform frequency-domain representations, for the nonuniform TLO formats, the aggregation is performed at the corresponding (time domain) referent impulse responses. Their frequency-domain representations are then subsequently produced and appropriately positioned as in the case of the Nu-FBMC counterpart. Spectrum of the even nonuniform TLO FBMC arrangement and its corresponding time-domain representation are shown in Figure 13a, b. Notice that the central frequencies are the same as in the case of Nu-FBMC-FLO arrangements depicted in Figure 10a and Figure 11a. Roll-off factors defined in time-domain are $100 \%, 50 \%, 50 \%, 25 \%, 25 \%, 12.5 \%$, and $6.25 \%$, respectively, starting from the narrowest (black) subchannel to the widest (cyan) subchannel (Figure 13b).

Figure 14 illustrates the matching of transmitted and received data for even $\mathrm{Nu}$-FBMC-TLO arrangements depicted in Figure 13a. Received data are reconstructed with MSE $\sim 5{ }^{\prime \prime} 10^{-6}$.

It can be concluded that the MSE values in all above observed cases are very small, and the scattering around the nominal values on constellation diagrams is insignificant.

\subsection{Concept of aggregation and selected configurations} Similar to Section 4.2 in which orthogonality for nonuniform FLO formats was derived based on the uniform
FLO orthogonality conditions; the orthogonality of the nonuniform TLO formats can be reduced to the uniform TLO case.

The orthogonality conditions of the uniform TLO FBMC format was derived in [9] by applying the timefrequency duality, i.e., by formally replacing symbols $\hat{G}$, $\omega, F$, and $T$ from (7) by $g, t, f$, and $2 \pi / T$, respectively. Based on the time-frequency duality between FLO and TLO, expression (12) for nonuniform FLO FBMC is also applicable to the nonuniform TLO FBMC case, with appropriately interchanged variables.

Derivation is given below only for the simplest case, where one subchannel has two times longer signaling interval compared to its adjacent one, as illustrated in Figure 15, without loss of generality.

If the referent impulse responses of uniform subchannels from Figure 15a are $g_{n-1, k}(t), g_{n, k}(t)$, and $g_{m, l}(t)$, obviously $m=n+1$, and the impulse response of the double wide subchannel $N$ from Figure 15b is $g_{N, K}(t)$. The process of time-domain aggregation is expressed through the following:

$$
g_{N, K}(t)=\sqrt{g_{n-1, k}^{2}(t)+g_{n, k}^{2}(t)}
$$

By placing the newly generated impulse responses (13) into (2), it follows as:

$$
\begin{aligned}
\operatorname{Re} & \left\{\int_{-\infty}^{\infty} g_{N, K}(t) g_{m, l}^{*}(t) d t\right\} \\
= & \operatorname{Re}\left\{\int_{-\infty}^{\infty} \sqrt{g_{n-1, k}^{2}(t)+g_{n, k}^{2}(t)} g_{m, l}^{*}(t) d t\right\} \\
= & \operatorname{Re}\left\{\int_{-\infty}^{\infty} \sqrt{g_{n-1, k}^{2}(t) g_{m, l}^{* 2}(t)+g_{n, k}^{2}(t) g_{m, l}^{* 2}(t)} d t\right\}
\end{aligned}
$$

Due to the absence of overlapping among the uniform TLO subchannels' impulse responses separated by (at 
a

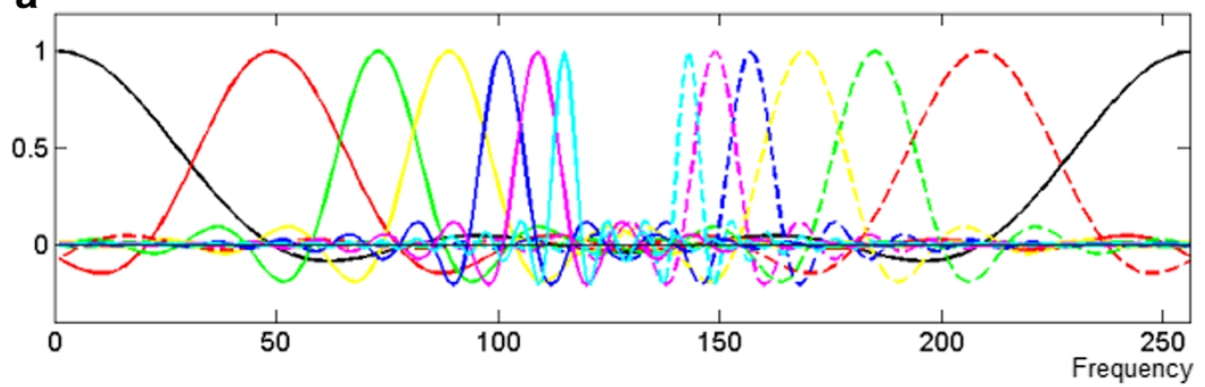

b

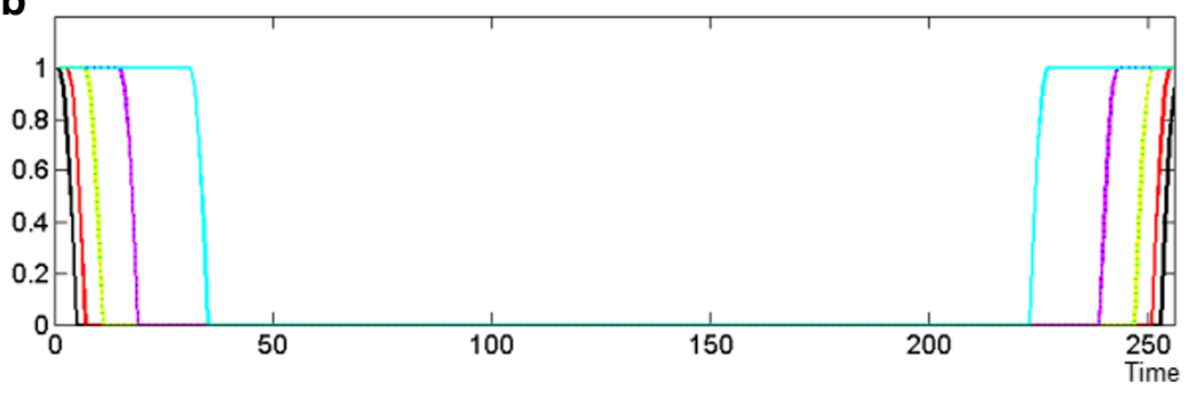

Figure 13 Nu-FBMC-TLO arrangements (a) and his corresponding time-domain representation (b).

least) one - the shortest - impulse response, the product $g_{n-1, k}^{2}(t) g_{m, l}^{* 2}(t)$ is equals 0 , so that the orthogonality criterion is reduced to the one corresponding to the uniform FBMC-TLO case.

\section{Spectral efficiency with HPA}

Considering the new emerging wireless technology, spectral and power efficiency are essential in the context of

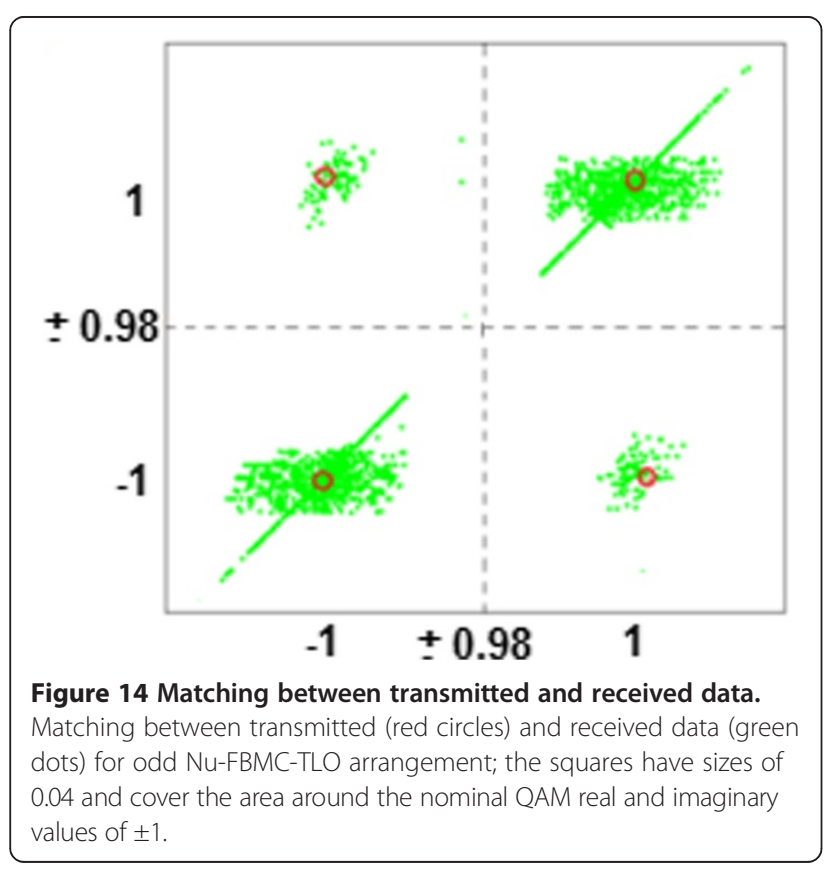

efficient utilization of spectrum under the adjacent channel interference avoidance constraints. The conventional CP-OFDM does not fulfill these demands for future wireless technologies. On the other hand, FBMC formats are expected to be more suitable in that sense.

Comparison of different FBMC formats and conventional CP-OFDM through the IEEE 802.11a standard PSD mask fitting are shown below. The LTE-related analysis that covers a wider frequency range around the nominal bandwidth are provided in deliverable D3.3, Section 4, www.ict-emphatic.eu.

Figure 16 shows the comparison between conventional CP-OFDM, uniform FLO, and uniform TLO configurations in the context of sensitivity to the nonlinear amplification (HPA). Power spectral densities are given for undistorted and distorted (with the Rapp's HPA model parameter $p=2.5$ [11]) conventional CP-OFDM with 52 active out of 64 subchannels (81.0\% of spectral efficiency, including four pilots), uniform FLO FBMC with seven out of eight subchannels, and uniform TLO FBMC, also with seven out of eight subchannels, which corresponds to spectrum usage of $87.5 \%$.

From Figure 16, it can be seen that the uniform FLO configuration provides even slightly less out-of-band leakage in comparison with CP-OFDM and therefore causes less interference allowing better utilization of gaps in the spectrum. The uniform TLO configuration shows similar behavior like CP-OFDM, except for frequencies being well outside the passband. The uniformly spaced FBMC thus has by approximately $6.5 \%$ better spectral efficiency than the CP-OFDM and even provides better PSD mask fitting. 

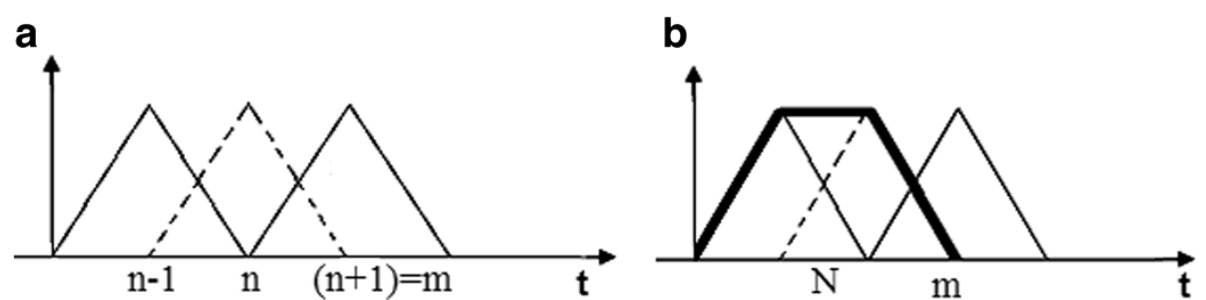

Figure 15 Uniform and nonuniform configurations. (a) Uniform configuration and its corresponding (b) nonuniform configuration for two subchannels.

Similar comparison is given in Figure 17 for symmetrical and asymmetrical Nu-FBMC-FLO with 58 out of 64 constituent uniformly spaced subchannels and the $\mathrm{Nu}$-FBMC-TLO with the same available bandwidth utilization (corresponding Nu-FBMC arrangements are depicted in Figure 10a, Figure 11a, and Figure 13a, respectively). The actual number of subchannels in all these cases was 13, and their bandwidths are progressively halved starting from DC-positioned one.

From Figure 17, it can be seen that the nonuniform FBMC configurations have better spectral features and provide better PSD mask fitting than the corresponding uniform FBMC configurations they were derived from. At the same time, nonuniform configurations provide a higher spectral efficiency of $91 \%$. Also, it can be seen that the asymmetrical FBMC FLO configuration is just slightly better than the symmetrical one in the context of reduction of spectral spread-out, which corroborates the expectations made in Section 5. The reason that this improvement is not that significant could be found in relation to discussion at the very end of Section 5, namely, the largest relative shortening of the impulse responses takes place at the relatively wide subchannels that are positioned close to DC.

From the presented results, it can be concluded that the nonuniform FBMC configurations offer better reduction of spectral spread-out in the case of nonlinear amplification than the uniform FBMC configurations. This could suggest that nonuniform configurations would provide a better reduction of PAPR as well; however, simulation results do not support this. In order to evaluate the PAPR features of analyzed uniform and nonuniform FBMC configurations, simulations are performed and results are given in Figure 18 in the form of PAPR complementary cumulative distribution function (CCDF).

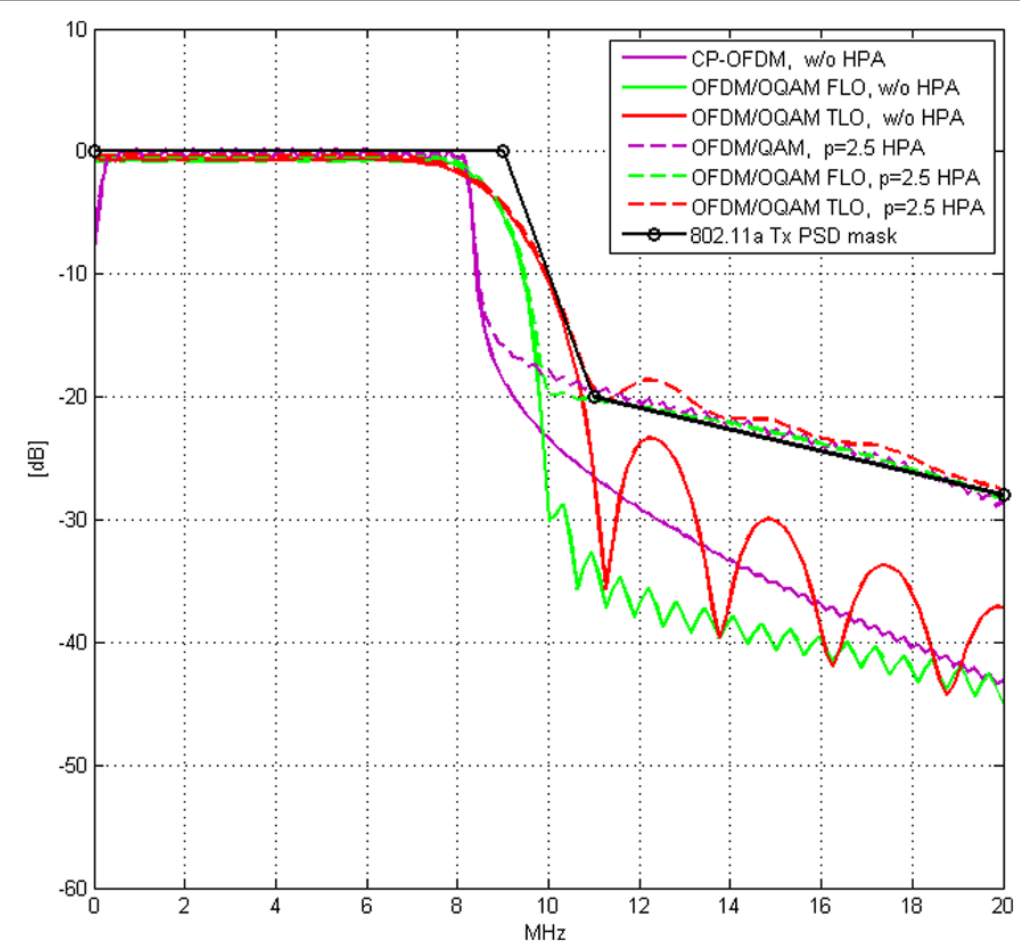

Figure 16 Comparison of CP-OFDM, FLO FBMC, and TLO FBMC. Comparison of conventional CP-OFDM, uniform FLO FBMC, and uniform TLO FBMC in context of HPA and mask fitting. 


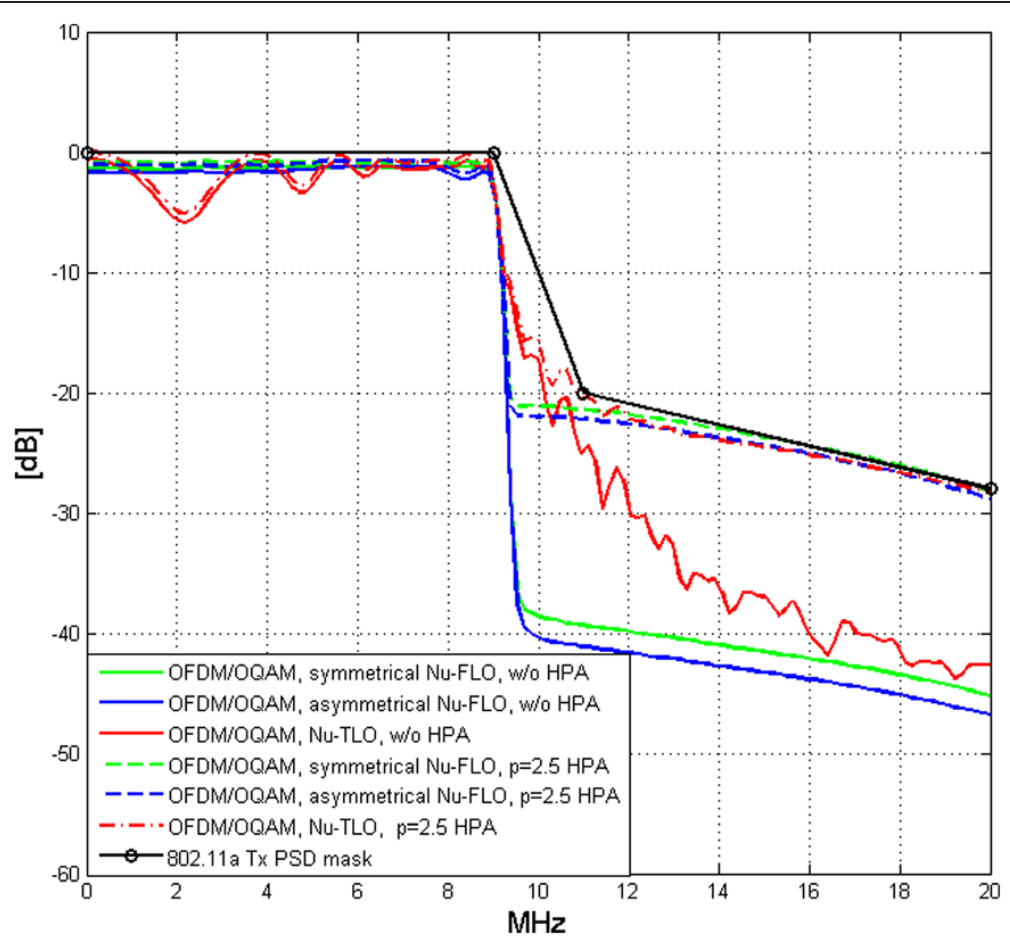

Figure 17 Comparison of symmetrical and asymmetrical Nu-FLO FBMCs and Nu-TLO FBMC. Comparison of symmetrical Nu-FBMC-FLO, asymmetrical Nu-FBMC-FLO and Nu-FBMC-TLO in context of HPA and mask fitting; the PSD variation in the pass-band region is related to nonsymmetry of the subchannels spectra for the Nu-FBMC-TLO case.

From Figure 18, it can be seen that all FBMC configurations (uniform and nonuniform, FLO and TLO) provide better PAPR reduction performances than it is the case with the standard CP-OFDM, which is consistent with spectral characteristics depicted in Figures 16 and 17.
When we compare uniform FLO and uniform TLO configurations, it is noticeable that the uniform TLO configuration provides lower PAPR CCDF values, which is expected, because TLO uses shorter impulse response. On the other hand, the longer impulse responses of narrower

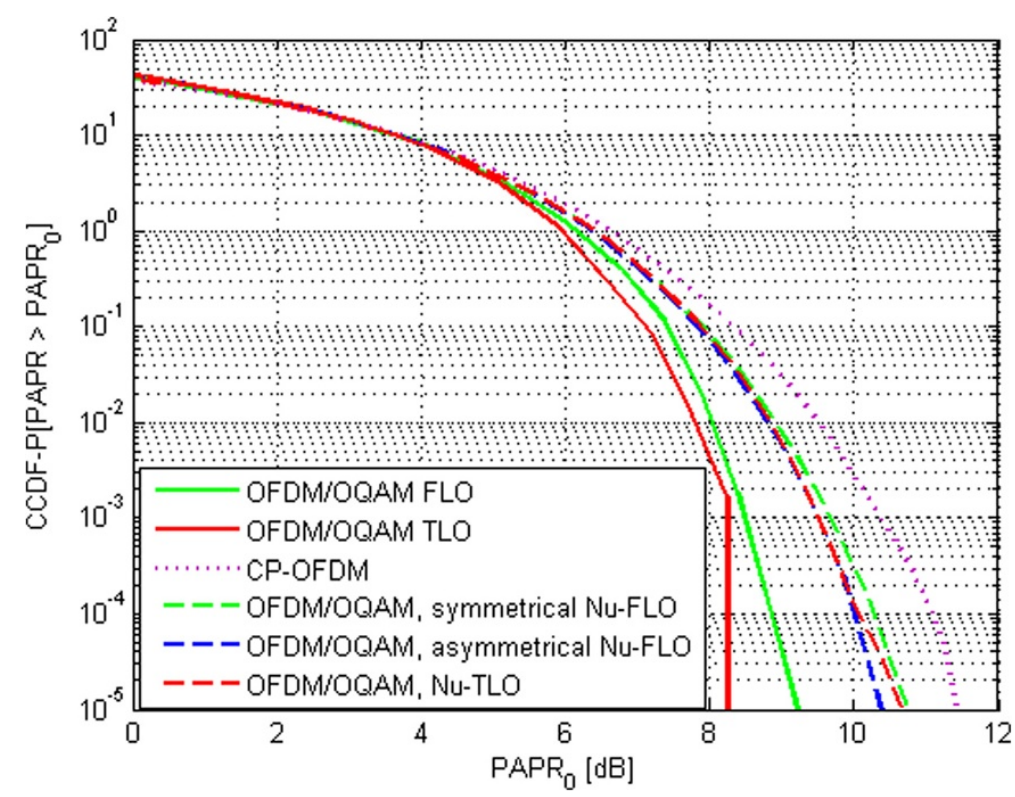

Figure 18 PAPR complementary cumulative distribution function, where $\mathrm{PAPR}_{0}$ designates the threshold. 
subchannels in nonuniform configurations (dashed curves on Figure 18) increase PAPR with respect to the corresponding uniform configurations (solid curves on Figure 18). It can also be seen that the asymmetrical configuration is slightly better above $10 \mathrm{~dB}$ than the symmetrical one which is in accordance with the expectations discussed in Section 5 and the results depicted in Figure 17.

\section{Comparison with conventional approaches}

The field of the nonuniform filter banks analysis for the source coding applications is fairly well developed, and two basic configurations have emerged - modifications of the uniform filter banks implementation by combination of (I)FFT and PNs by appropriately optimizing the referent low-pass filter impulse response [12,13] and the application of quadrature-mirror filters based branching or tree architectures [14]. While the first case the roll-off factors remain relatively high, the tree-branching method keeps the same roll-off factor for all subchannels, with maximal value inversely proportional to the number of nonuniform subchannels. While these two structures may have similar complexity of implementation, their flexibility appears to be much smaller.

\section{Conclusions}

We have presented a rather pragmatic approach for an extension of the uniformly spaced FLO and TLO FBMC formats towards the nonuniform ones. The paper provides the analysis and elaboration of nonuniform filter banks, with derivation of their orthogonality conditions.

The nonuniform FBMC framework can be of practical use as an element of a flexible channelization and, in particular, for the realization of communication channels for individual users. In the downlink, the asymmetrical spectral shaping removes the necessity for separation of adjacent users by frequency guard bands even if they individually use subchannels of different bandwidth. For the uplink directions, by deploying the nonuniform FBMC, the channels of individual users can be separated by a reduced loss in spectral efficiency, e.g., to accommodate PSD mask constraints, as demonstrated in Section 7.

The advantages of the relatively wide subchannels compared to those in the CP-OFDM regarding the robustness against frequency offset and phase jitter are implicit. The same applies to the influence of Doppler spread, provided that the channel variations can be tracked.

The potential resilience of the staggered I/Q formats and the communication (multicarrier) waveforms based on them against the (kindred) co-channel interference has yet to be fully explored in terms of the per subchannel equalization in the WLF context $[15,16]$. The linear and nonlinear (DFE) MMSE equalization is directly applicable to the nonuniformly spaced subchannels of the FLO kind, while for the uniform and nonuniform TLO formats, the adequate oversampling at the output of the analysis filter bank might be needed. The alternative successive interference cancelation (SIC) framework proposed in [17] for the FLO case is straightforwardly applicable by just increasing the number of adjacent subchannels to be taken into account.

\section{Endnote}

${ }^{a}$ From implementation and applicability point of view, the fast-convolution filter bank framework [18] will likely be a better alternative.

\section{Competing interests}

The authors declare that they have no competing interests.

\section{Acknowledgements}

This work was supported by an FP7grant, project EMPhAtiC, and ICT-318362 (http://www.ict-emphatic.eu/). The authors thank the reviewers for their numerous useful comments and suggestions, which very much improved the paper.

Received: 2 December 2013 Accepted: 6 November 2014 Published: 26 November 2014

\section{References}

1. P Amini, R Kempter, RR Chen, L Lin, B Farhang-Boroujeny, Filter bank multitone: a physical layer candidate for cognitive radios, in 2005 Software Defined Radio Technical Conference, 2005, pp. 14-18

2. $\quad \mathrm{P}$ Chevalier, F Pipon, New insights into optimal widely linear array receivers for the demodulation of BPSK, MSK, and GMSK signals corrupted by noncircular interferences - application to SAIC. Signal Process. IEEE Trans. 54(3), 870-883 (2006)

3. A Mirbagheri, KN Plataniotis, P Subbarayan, An enhanced widely linear CDMA receiver with OQPSK modulation. Commun. IEEE Trans. 54(2), 261-272 (2006)

4. M Konrad, W Gerstacker, Interference robust transmission for the downlink of an OFDM-based mobile communications system. Eurasip J. Wirel. Comm. 2008, 15 (2008)

5. B Le Floch, M Alard, C Berrou, Coded orthogonal frequency division multiplex [TV broadcasting]. Proc. IEEE 83.6, 982-996 (1995)

6. Z Cvetkovic, JD Johnston, Nonuniform oversampled filter banks for audio signal processing. IEEE Trans. Speech Audio Process. 11(5), 393-399 (2003)

7. A Eghbali, $\mathrm{H}$ Johansson, P Lowenborg, Reconfigurable nonuniform transmultiplexers using uniform modulated filter banks. Circuits Syst. I Regul. Pap. IEEE Trans. 58(3), 539-547 (2011)

8. M Bellanger, D Le Ruyet, D Roviras, M Terré, J Nossek, L Baltar, Q Bai, D Waldhauser, M Renfors, T Ihalainen, A Viholainen, TH Stitz, J Louveaux, A Ikhlef, V Ringset, H Rustad, M Najar, C Bader, M Payaro, D Katselis, E Kofidis, L Merakos, A Merentitis, N Passas, A Rontogiannis, S Theodoridis, D Triantafyllopoulou, D Tsolkas, D Xenakis, M Tanda, FBMC physical layer: a primer. PHYDYAS, (2010). http://www.ict-phydyas.org

9. R Li, G Stette, Time-limited orthogonal multicarrier modulation schemes. Commun. IEEE Trans. 43(234), 1269-1272 (1995)

10. RE Crochiere, LR Rabiner, Multirate digital signal processing, in ed. by AV Oppenheim. Pretice Hall Signal Processing Series, 1983, pp. 127-192

11. C Rapp, Effects of HPA-nonlinearity on a 4-DPSK/OFDM-signal for a digital sound broadcasting signal, in ESA, Second European Conference on Satellite Communications (ECSC-2), 1991, pp. 179-184. (SEE N92-15210 06-32), vol. 1, pp. 179-184

12. J Princen, The design of nonuniform modulated filterbanks. Signal Process. IEEE Trans. 43(11), 2550-2560 (1995)

13. SC Chan, XM Xie, TI Yuk, Theory and design of a class of cosine-modulated non-uniform filter banks. IEEE Int. Conf. Acoust. Speech Signal Process. 1, (2000). ICASSP'00. Proceedings

14. E Elias et al., Tree-structured IIR/FIR octave-band filter banks with very low-complexity analysis filters. Int. Symp. Circuits Syst. 2, (2001). ISCAS 2001. IEEE, 2001

15. S Nedic, An unified approach to equalization and echo cancellation in OQAM-based multi-carrier data transmission. Glob. Telecomm. Conf. 3, (1997). GLOBECOM'97., IEEE, 1997 
16. DS Waldhauser, Multicarrier Systems Based on Filter Banks. Shaker Verlag, Ph. D. Thesis (Technical University Munich, 2009)

17. M Aoude, R Vallet, S Nedic, Interference cancellation in coded OFDM/ OQAM. Int. Symp. Wirel. Commun. Syst. (ISWCS) (2012). IEEE, 2012

18. J Yli-Kaakinen, M Renfors, Fast-convolution filter bank approach for non-contiguous spectrum use. Future Netw. Mobile Summit (FutureNetworkSummit) (2013). IEEE, 2013

doi:10.1186/1687-6180-2014-167

Cite this article as: Jošilo et al:: Multicarrier waveforms with I/Q

staggering: uniform and nonuniform FBMC formats. EURASIP Journal on Advances in Signal Processing 2014 2014:167.

Submit your manuscript to a SpringerOpen ${ }^{\circ}$ journal and benefit from:

- Convenient online submission

- Rigorous peer review

- Immediate publication on acceptance

- Open access: articles freely available online

- High visibility within the field

- Retaining the copyright to your article

Submit your next manuscript at $\gg$ springeropen.com 